\title{
New Infrared Diffuse Interstellar Bands in the Galactic Center and Elsewhere
}

\author{
T. R. Geballe \\ Gemini Observstory, 670 N. A'ohoku Place, Hilo, HI 96720 U.S.A. \\ email: tgeballe@gemini.edu
}

\begin{abstract}
This paper updates the recent discovery of over a dozen new diffuse interstellar bands (DIBs), first in $H$-band spectra of stars in the Galactic center (GC) and toward stars in the Cygnus OB2 Association. The $H$-band DIBs, which currently number 15 , are the longest wavelength DIBs reported to date and are the first found on sightlines toward the Galactic center. $K$-band (2.0-2.5 $\mu \mathrm{m})$ spectra of the GC stars do not reveal additional DIBs. Comparison of the velocity profile of the strongest of the new DIBs in the sightline toward GCS3-2 (in the GC) with that toward Cygnus OB2 No. 9 and also with the broad velocity profiles of $\mathrm{H}_{3}^{+}$lines toward GCS3-2 confirm that a significant fraction of the diffuse material producing the DIB absorptions on sightlines to the GC is located within the central few hundred parsecs of the Galaxy.
\end{abstract}

Keywords. astrochemistry, ISM: lines and bands, Galaxy: center

\section{Introduction}

A number of diffuse interstellar bands (DIBs) were recently discovered in the infrared $H$-band $(1.5-1.8 \mu \mathrm{m})$ on sightlines toward objects in the Galactic center (GC) by Geballe et al. (2011). Most of the $H$-band DIBs are intrinsically weak but are observable toward the GC because of the large visual extinction ( $\sim 30 \mathrm{mag})$, which is believed to be mostly due to dust in diffuse clouds (Whittet et al. 1997). The strongest by far of the newly discovered DIBs is a band centered at $1.527 \mu \mathrm{m}$; its peak optical depth is 0.2 , at least three times larger than any of the others. Although these DIBs had not been reported previously toward diffuse clouds in the Galactic plane, examination of unpublished spectra toward stars in the Cygnus OB2 association revealed the presence of the stronger $H$-band DIBs (Geballe et al. 2011). They have also been detected toward a number of additional Galactic sources (Cox et al. this volume). The $H$-band DIBs are the longest wavelength DIBs currently known. Prior to their discovery the longest wavelength DIB known was at $1.318 \mu \mathrm{m}$ (Joblin et al. 1990).

\section{Current Census}

In total $15 H$-band DIBs have now been found. They are shown in Fig. 1, which contains a spectrum of the Quintuplet star GCS4 (Nagata et al. 1990, Okuda et al. 1990), also know as Q3 (Moneti, Glass, \& Moorwood 1994, Figer et al. 1999). The most recently discovered of the bands are a narrow feature at $1.485 \mu \mathrm{m}$ (at the short wavelength edge of the $H$-band) and a broad feature at $1.585 \mu \mathrm{m}$; they are the only ones not reported by Geballe et al. (2011). Here, by an individual DIB we refer to a single absorption maximum. Some of the the bands, in particular the triplet near $1.57 \mu \mathrm{m}$ and the triplet near $1.66 \mu \mathrm{m}$, not easily seen in Fig. 1 but apparent in Fig. 2 of Geballe et al. (2011), are close to one another in wavelength and could be regarded as a multiplet DIB rather than 
multiple DIBs. A few additional weak absorption features are present in this spectrum, but have not been conclusively identified in spectra of other GC sources.

Many of the spectra of Galactic center objects in which the $H$-band DIBs have been found extend through the $K$ band $(2.0-2.5 \mu \mathrm{m})$. No $K$-band DIBs have been detected in those spectra, to central optical depth limits of $\sim 0.02$. Most of the $H$-band DIBs are considerably stronger than that. This shows that there is a significant drop-off in DIB strength with increasing wavelength from the $H$-band to the $K$-band and continues the trend between the $J$ and $H$ bands. Based on the spectra of both the Galactic center objects and Cygnus OB2 stars, and using a diffuse cloud extinction of 20 visual magnitudes toward the GC sources (Whitet et al. 1997) the strongest $H$-band DIB, at $1.527 \mu \mathrm{m}$, has an equivalent width of $60 \mathrm{~m} \AA$ per magnitude of extinction, less than half that of the stronger of the two known $J$-band DIBs (Joblin et al. 1990).

\section{Current research}

Our current efforts are directed toward analyzing very recently obtained high resolution $\left(\mathrm{R}=18,000\right.$, corresponding to a velocity resolution of $17 \mathrm{~km} \mathrm{~s}^{-1}$ ) spectra of the $H$ band DIBs toward a number of sources in the Galactic plane whose radial velocities are

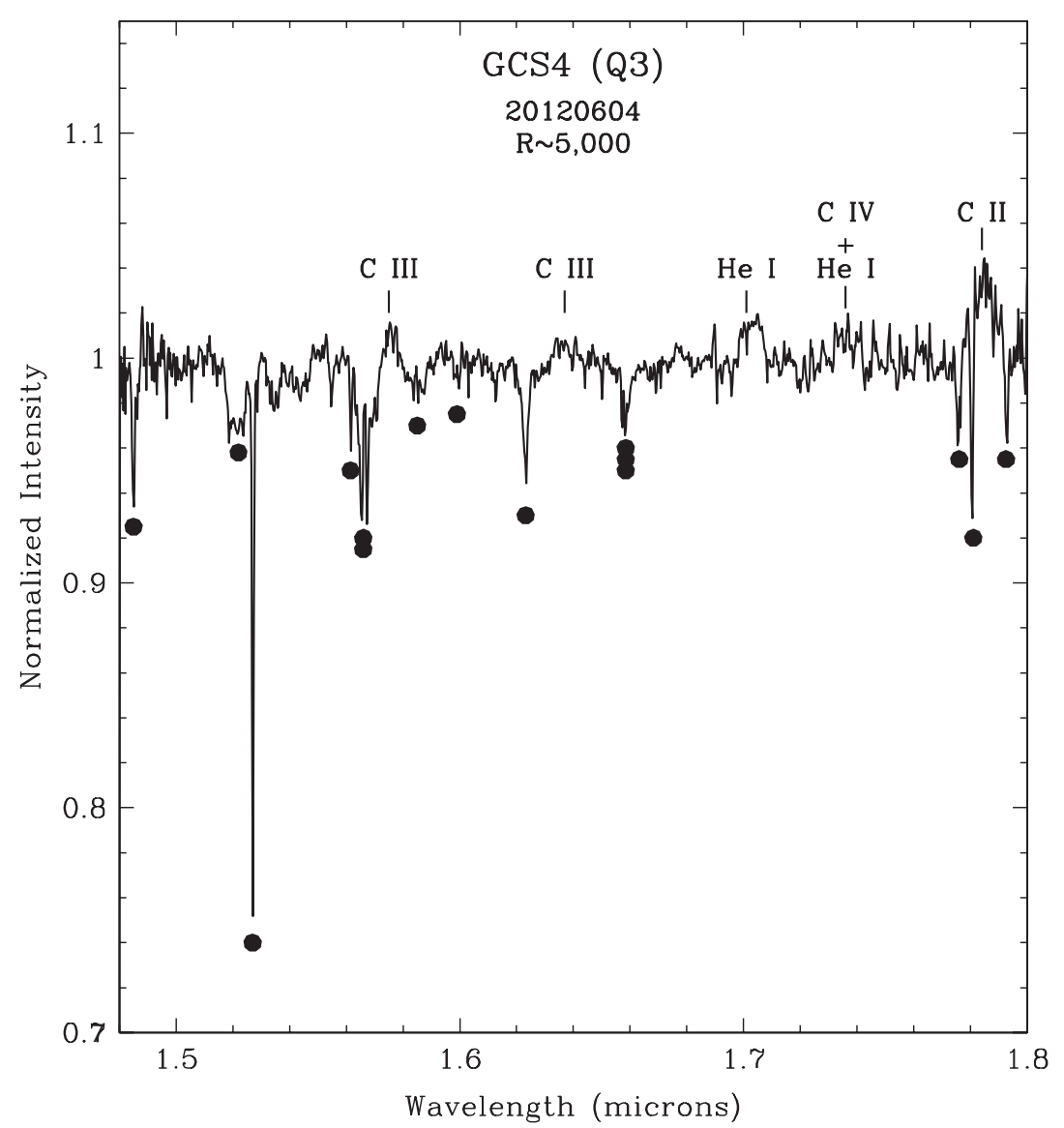

Figure 1. H-band spectrum of the Quintuplet source GCS4. The diffuse interstellar bands are denoted by large dots. Emission lines in the star are also identified. 
accurately known. This will allow the determination of accurate laboratory wavelengths for the DIBs. In addition, we are obtaining a high-resolution spectrum of the strong and narrow $1.527 \mu \mathrm{m}$ DIB toward one object in the Galactic center, the Quintuplet source GCS3-2 (Q2). GCS3-2 lies within the Central Molecular Zone (CMZ), a region of radius 200 pc. Spectra of $\mathrm{H}_{3}^{+}$toward it and other Galactic center sources have revealed a large quantity of warm and diffuse gas with LSR velocities between 0 and $-150 \mathrm{~km} \mathrm{~s}^{-1}$ within the CMZ (Oka et al. 2005). The gas at high negative velocities dispersion should appear as a blue-shifted wing on the profile of the $1.527 \mu \mathrm{m}$ DIB.

Figure 2 shows preliminary spectra of Cygnus OB2 No. 9 and GCS3-2 in the $1.527 \mu \mathrm{m}$ DIB, on a common laboratory wavelength scale (wavelengths are in vacuum). We have used a value of $6.5 \mathrm{~km} \mathrm{~s}^{-1}$ as the LSR velocity of the Cygnus OB2 diffuse cloud, obtained from the $\mathrm{H}_{3}^{+}$spectra of McCall et al. (2005). From Cygnus OB2 No. 9 we determine a preliminary rest wavelength for this DIB of $(1.52722 \pm 0.00005) \mu \mathrm{m}$ and an intrinsic FWHM (after correcting for the instrumental resolution and the velocity dispersion of the cloud as observed in $\left.\mathrm{H}_{3}^{+}\right)$of $(0.00042 \pm 0.00006) \mu \mathrm{m}$. These values will be refined after all of the data are obtained and combined.

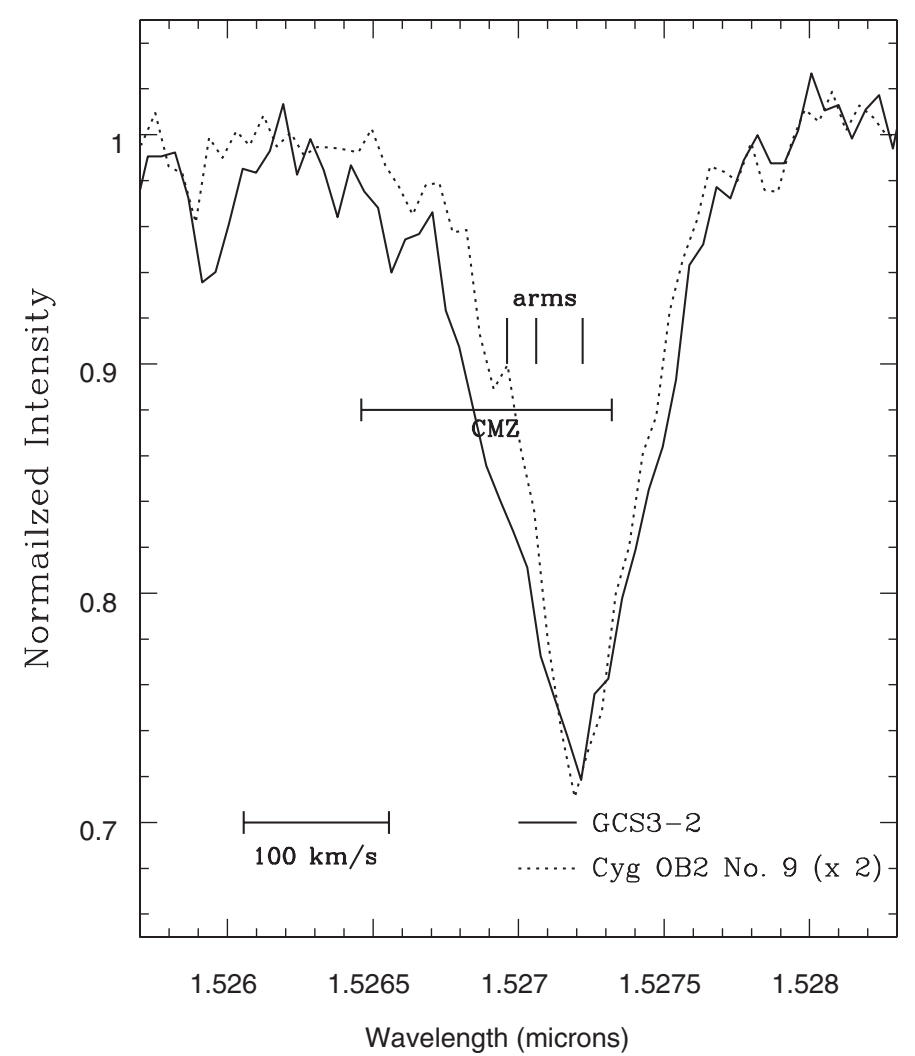

Figure 2. Spectra of the $1.527 \mu \mathrm{m}$ DIB in Cygnus OB 2 No. 9 and the Galactic center Quintuplet source GCS3-2 at a resolution of $17 \mathrm{~km} \mathrm{~s}^{-1}$. The wavelength scale has been adjusted to be in laboratory microns for Cygnus OB2 No. 9 (by correcting for the earth's orbital velocity and the radial velocity of the Cygnus OB2 diffuse cloud, and thus yields the rest wavelength of this DIB. The spectrum of GCS3-2 has been shifted into the same reference frame. The vertical lines indicate the velocities where there may be contributions to the GCS3-2 spectrum from foreground Galactic arms at $0,-32$, and $-52 \mathrm{~km} \mathrm{~s}^{-1}$. The nearby horizontal line denotes velocity range of the warm and diffuse gas in the CMZ observed by Oka et al. (2005). 
In addition to the diffuse gas in the $\mathrm{CMZ}$, diffuse gas in the foreground Galactic arms near $0,-30$, and $-50 \mathrm{~km} \mathrm{~s}^{-1}$ likely contributes to the absorption profile obseved toward GCS3-2. However, the extended blue-shifted wing on the profile indicates that a significant contribution to the profile is also made by the higher negative velocity CMZ gas. We expect to use this absorption profile to determine the relative contributions of the arms and the CMZ. If the abundance of carrier of this DIB is mainly determined by the abundance of carbon, the spectrum should also provide a constraint on the carbon abundance in the CMZ, which some evidence suggests is considerably higher than solar (Sodroski et al. 1995).

\section{Scientific Value}

The ultimate aim of much DIB science is to identify the carriers of these bands, an endeavor that has frustrated astrophysicists and astrochemists for nearly a century. Nevertheless, astronomers should find the infrared DIBs useful for their own purposes, as illustrated in the preceding section. Since they are observable toward regions of high extinction, the infrared DIBs should be of assistance in studying the diffuse interstellar medium in obscured regions, either in our galaxy or in extragalactic objects. Clusters of hot and massive stars in distant regions of the Galaxy, could be prime candidate probes for such studies, as they are luminous and some stars in them have relatively featureless spectra, a near necessity for qualitatively measurements of these bands.

Regarding identification, several unique features of the infrared DIBs may be advantages over shorter wavelength DIBs and may assist in their ultimate identification, as follows.

- Their longer wavelengths may constrain the type of radiative transition and the nature of the carrier.

- Their presence in a less congested part of the spectrum may assist in ruling out candidate carriers.

- The apparent cut-off in DIBs near $2.0 \mu \mathrm{m}$ may provide a useful constraint.

- Their presence in the GC, a harsher environment in terms of gas temperature and cosmic ray density than diffuse clouds far from the center, may rule out some fragile DIB candidate carriers.

\section{Acknowledgements}

I thank my colleagues, F. Najarro, D. F. Figer, D. de la Fuente, and B. W. Schlegelmilch for our fruitful collaboration.

\section{References}

Figer, D. F., McLean, I. S., \& Morris, M. 1999, ApJ, 514, 202

Geballe, T. R., Najarro, F., Figer, D. F., Schlegelmilch, B. W., \& de la Fuente, D. 2011, Nature, 479,200

Joblin, C., Maillard, J. P., d'Hendecourt, L., \& Leger, A. 1990, Nature, 346, 729

McCall, B. J., Hinkle, K. H., Geballe, T. R., et al. 2002 ApJ, 567, 391

Moneti, A., Glass, I. S., \& Moorwood, A. 1994, MNRAS, 268, 194

Nagata, T., Woodward, C. E., Shure, M., Pipher, J. L., \& Okuda, H. 1990, ApJ, 351, 83

Oka, T., Geballe, T. R., Goto, M., Usuda, T., \& McCall, B. J. 2005, ApJ, 632, 882

Okuda, H. et al. 1990 ApJ, 351, 89

Sodroski, T. J., Odegard, N., Dwek, E., et al. 1995 ApJ. 452, 262

Whittet, D. C. B., Boogert, A. C. A., Gerakines, P. A., et al. 1997, ApJ, 490, 729 